\title{
Odd Tensor Modes from Inflation
}

\author{
Lorenzo Sorbo \\ UMass Amherst
}

CMB Polarization, Minneapolis, 0I//4/20I5 
Scalar perturbations during inflation rich phenomenology:

- Features

- Isocurvature

- Non vacuum states

- Nongaussianities

- Oscillations

Tensors typically assumed to be boring....

$$
\mathcal{P}_{t} \propto \frac{H^{2}}{M_{P}^{2}}
$$

$H>$ during inflation slightly red spectrum 


\section{This talk:}

Non-boring

tensors 


\section{The system}

During inflation, a rolling pseudoscalar $\phi$ interacting with a $U(1)$ gauge field via

$$
\mathcal{L}_{\phi F F}=\frac{\phi}{f} \epsilon_{\alpha \beta \gamma \delta} F^{\alpha \beta} F^{\gamma \delta}
$$

( $f=$ constant with dimensions of a mass) 
The helicity- $\lambda$ mode functions $A_{\lambda}(k, \tau)$ are sourced by $\phi$

$$
A_{\lambda}^{\prime \prime}+\left(\mathbf{k}^{2}+\lambda \frac{\phi^{\prime}}{f}|\mathbf{k}|\right) A_{\lambda}=0
$$

for $\lambda=-$, the "mass term" is negative and large for $\sim 1$ Hubble time:

.Anber and LS 06

Exponential amplification of left handed modes only!

$$
\text { parity violation! }
$$

$$
A_{L} \propto \exp \left\{\frac{\pi}{2} \frac{\dot{\phi}}{f H}\right\}
$$




\section{Generation of parity violating, large amplitude gravitational waves}

The energy of the electromagnetic field sources gravitational waves of helicity- $\lambda h \lambda$ :

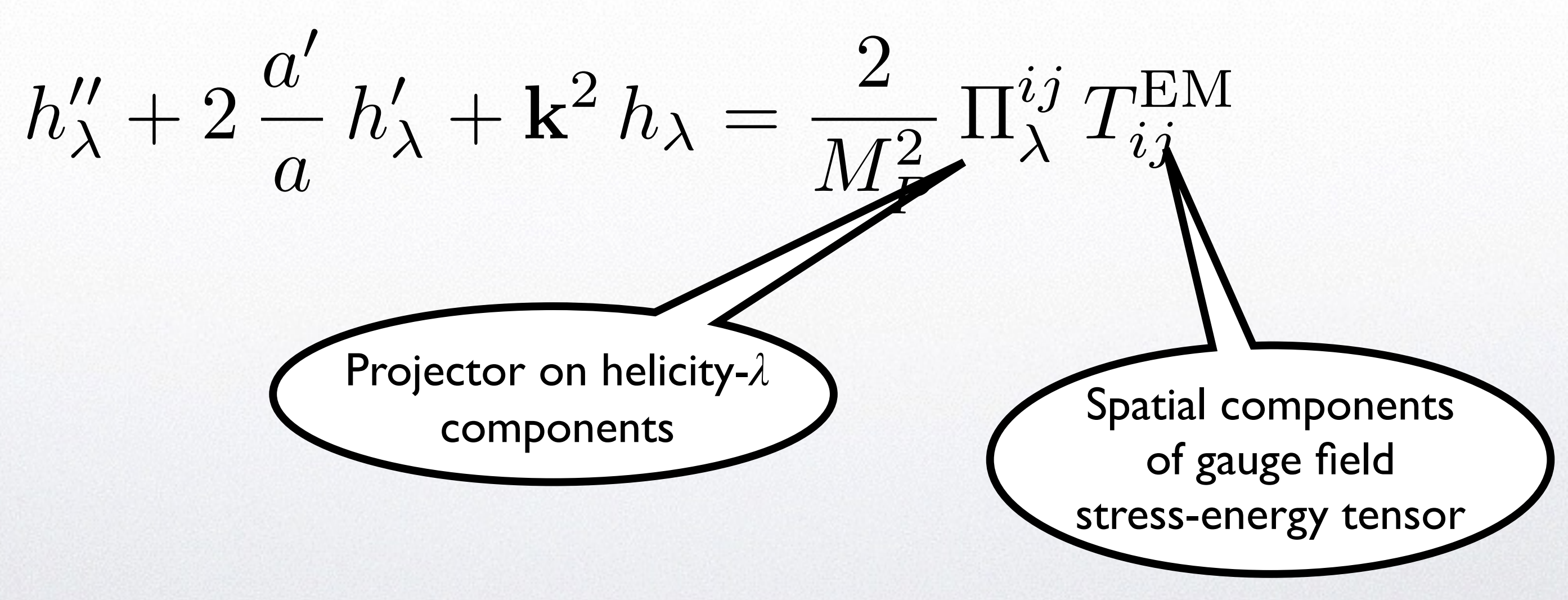




\title{
Parity violating gravitational waves
}

\author{
$A_{L}$ and $A_{R}$ have different amplitudes
}

$<h_{L} h_{L}>\neq<h_{R} h_{R}>$

Physics: in the limit of small transverse momentum two LH photons cannot create a $\mathrm{RH}$ graviton

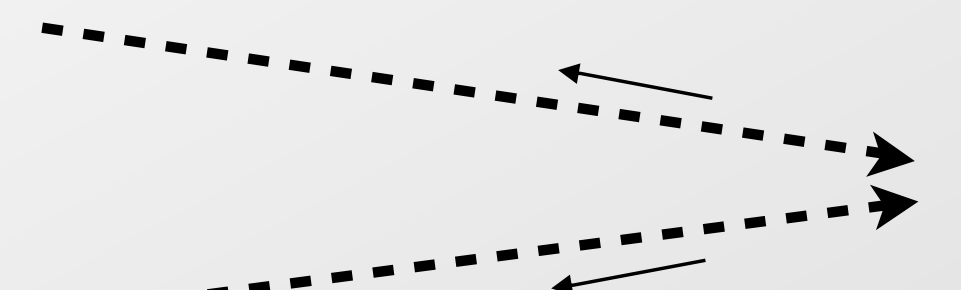




\section{The parity-violating power spectrum}

$$
\begin{gathered}
\mathcal{P}_{L}(\mathbf{k})=\frac{H^{2}}{\pi^{2} M_{P}^{2}} \\
\mathcal{P}_{R}(\mathbf{k})=\frac{H^{2}}{\pi^{2} M_{P}^{2}}\left(1+9 \times 10^{-} \frac{H^{2}}{M_{P}^{2}} \frac{e^{4 \pi \xi}}{\xi^{6}}\right) \\
\begin{array}{c}
\text { "standard" } \\
\text { parity-invariant part }
\end{array} \\
\text { parity-violation } \\
\xi
\end{gathered}
$$




\title{
How do we see the effect of parity violating GWs?
}

\author{
While $T$ and $E$ modes are parity-even, \\ $B$ is parity-odd
}

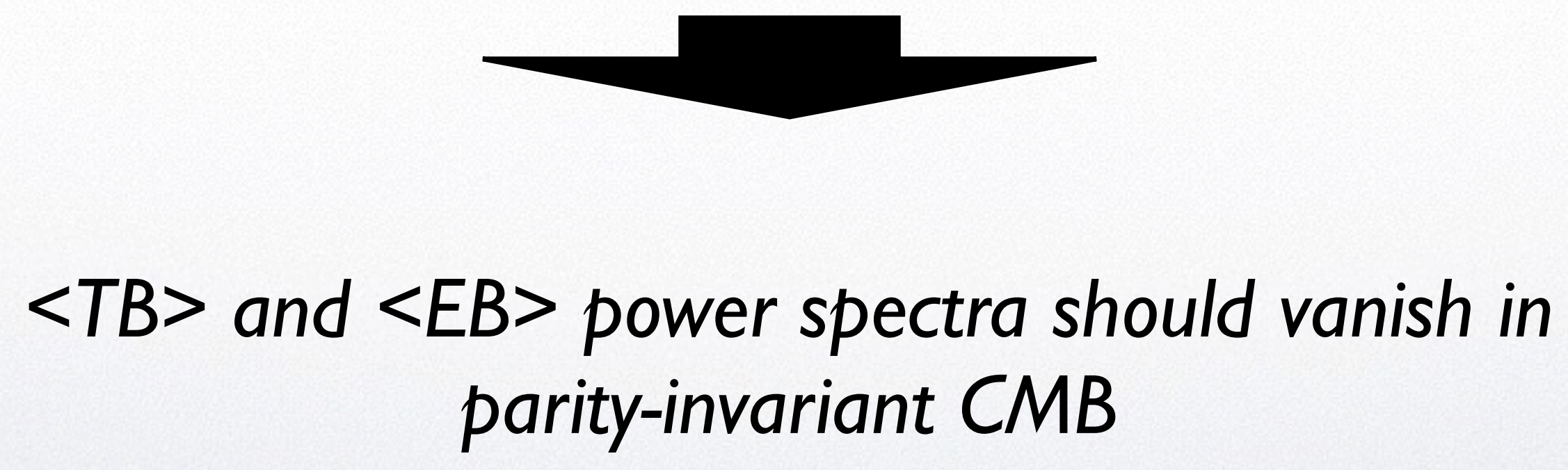




\section{Detection prospects related to observability}

\section{nonzero $\langle E B\rangle$ and/or $\langle T B\rangle$}

Saito Ichicki Taruya 07,

Contaldi Maguejio Smolin 08, Gluscevic Kamionkowski 10

Depend on two parameters

$$
r=\frac{\mathcal{P}_{R}+\mathcal{P}_{L}}{\mathcal{P}_{T}}
$$

tensor-to-scalar ratio

$$
\Delta \chi=\frac{\mathcal{P}_{R}-\mathcal{P}_{L}}{\mathcal{P}_{R}+\mathcal{P}_{L}}
$$

chirality of primordial perturbations

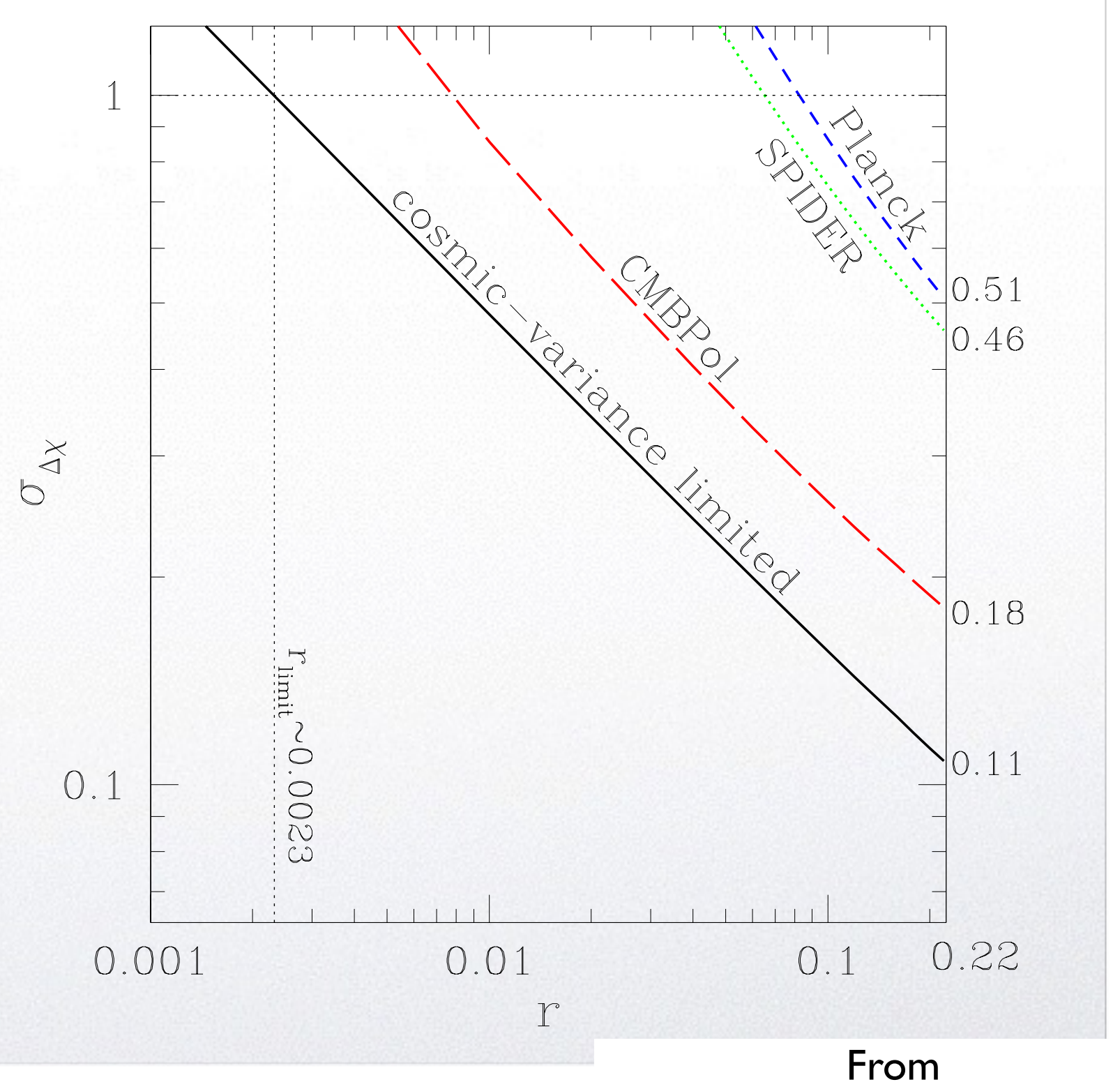




\section{Detection prospects related to observability of}

\section{nonzero $<E B>$ and/or $\langle T B>$}

(more recent analysis)

Ferte and Grain 14

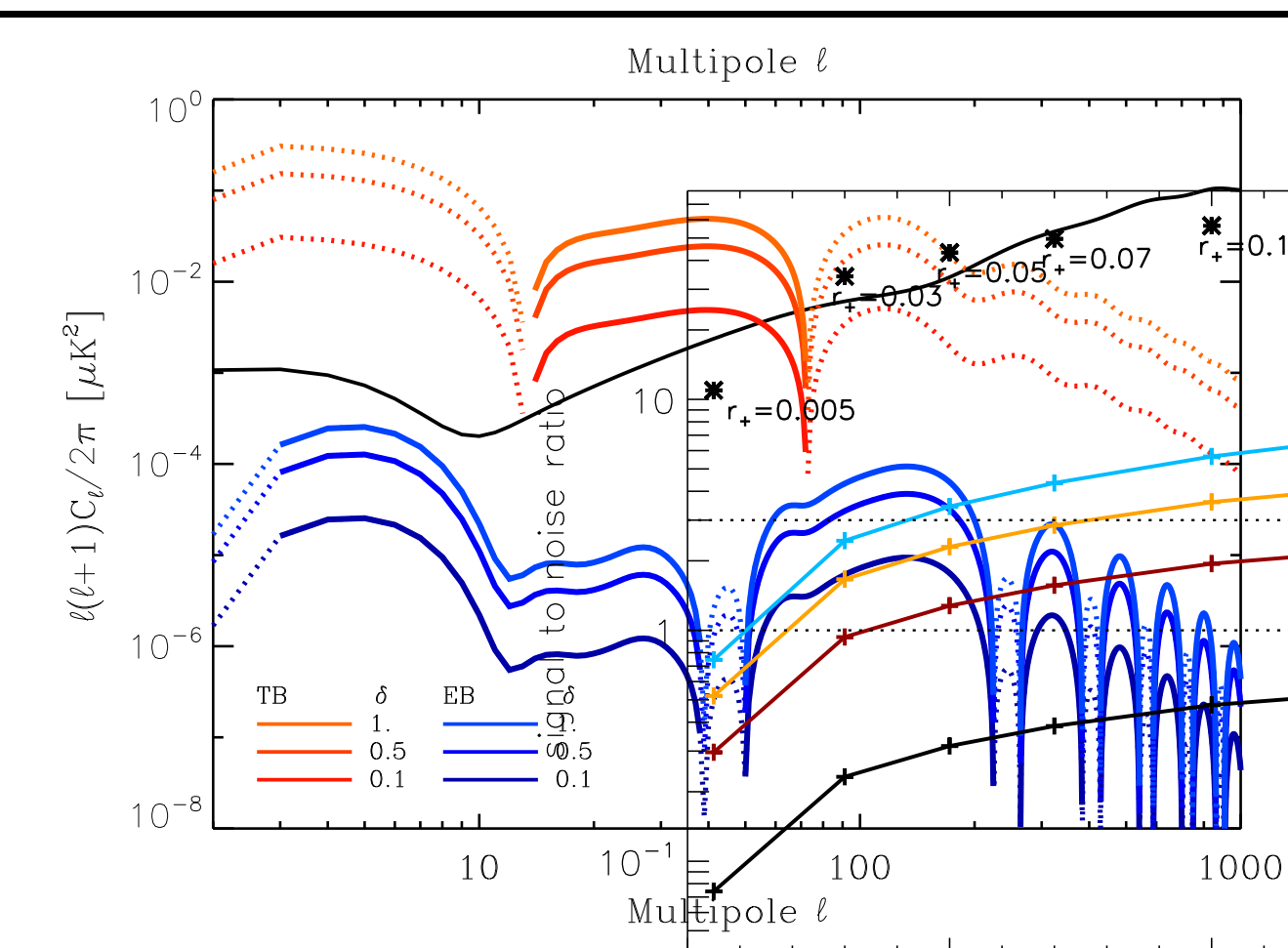

FIG. 1: Upper panel: Angular power spectra for primary CMB anisotropies for $B B$ (black curve), $T B$ (red curves) and $E B$ (blue curves) correlations. The parameters $r_{(+)}$is set equal to 0.05 and $\delta$ varies from 0.1 (meaning $10 \%$ of parity violation) to 1 ( $100 \%$ of parity violation). Solid lines correspond to positive values of the angular power spectra and dashed lines correspond to negative values. Changing from $(\delta)$ to $(-\delta)$ with $r_{(+)}$unchanged changes the sign of $C_{\ell}^{T B}$ and $C_{\ell}^{E B}$ and leaves $C_{\ell}^{B B}$ unaffected. We note that smaller $|\delta|$ translates into smaller $\left|r_{(-)}\right|$. Lower panel: Same as upper panel but taking into account the impact of gravitational lensing.

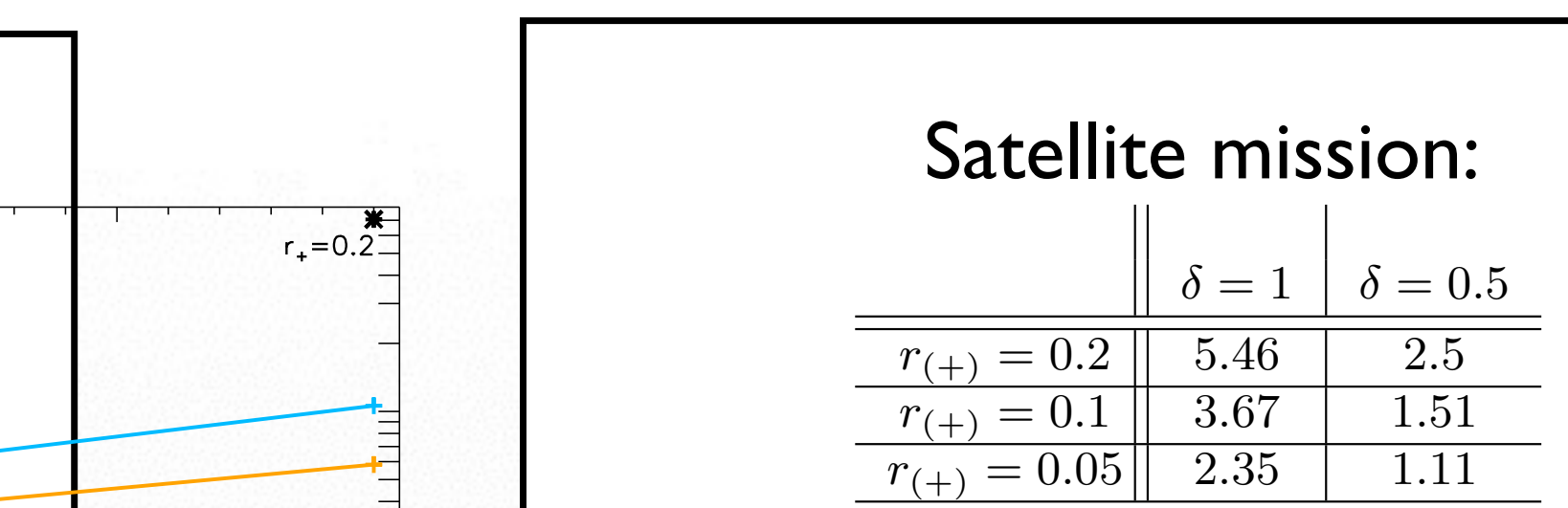

TABLE II: Signal-to-noise ratio on $r_{(-)},(\mathrm{S} / \mathrm{N})_{r_{(-)}}$, as derived from a pure pseudospectrum reconstruction of the angular power spectra. We remind that for a given value of $r_{(+)}$and $\delta$, the value of $r_{(-)}$is $r_{(-)}=\delta \times r_{(+)}$.

\section{Balloon/Ground}

\begin{tabular}{c||c|c|c|c|c|c}
\multicolumn{1}{r|}{$r_{(+)}$} & 0.2 & 0.1 & 0.07 & 0.05 & 0.03 & 0.007 \\
$r_{(-)}$ & & & & & & \\
\hline \hline 0.2 & 1.22 & & & & & \\
\hline 0.1 & 0.43 & 0.64 & & & & \\
\hline 0.07 & 0.29 & 0.4 & 0.487 & & & \\
\hline 0.05 & 0.2 & 0.28 & 0.326 & 0.38 & & \\
\hline 0.03 & 0.12 & 0.16 & 0.188 & 0.216 & 0.27 & \\
\hline 0.007 & 0.03 & 0.037 & 0.043 & 0.049 & 0.06 & 0.1 \\
\hline
\end{tabular}

TABLE III: Signal-to-noise on $r_{(-)}$for different values of $r_{(+)}$ in the case of small-scale (ballon-borne or ground-based) experiments, and using a mode-counting expression for the error bars on the angular power spectra reconstruction. 


\section{For our system

$$
\Delta \chi=\frac{4.3 \times 10^{-7} \frac{e^{4 \pi \xi}}{\xi^{6}} \frac{H^{2}}{M_{P}^{2}}}{1+4.3 \times 10^{-7} \frac{e^{4 \pi \xi}}{\xi^{6}} \frac{H^{2}}{M_{P}^{2}}} .
$$$$
\xi \equiv \frac{\dot{\phi}}{2 f H}
$$

Exponential dependence on the coupling $1 / f$

In principle sizable parity violation in large portion of parameter space.

Anything more? 


\section{Photons source metric perturbations}

in a $2 \rightarrow 1$ process

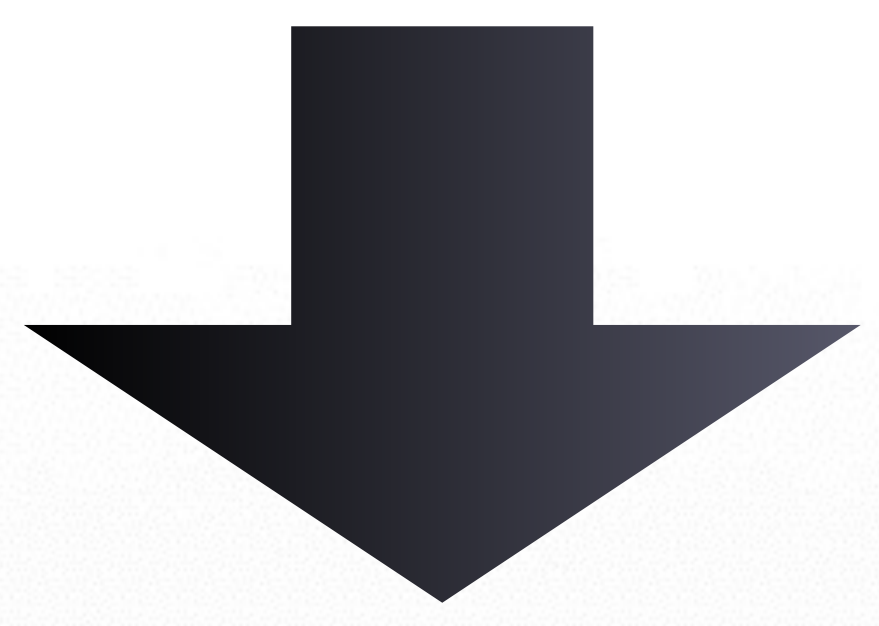

(equilateral) 


\section{Photons source metric perturbations in a $2 \rightarrow I$ process:}

$$
\left\langle\hat{h}_{-}\left(\mathbf{k}_{1}\right) \hat{h}_{-}\left(\mathbf{k}_{2}\right) \hat{h}_{-}\left(\mathbf{k}_{3}\right)\right\rangle_{\text {equil }}=6.1 \times 10^{-10} \frac{\delta\left(\mathbf{k}_{1}+\mathbf{k}_{2}+\mathbf{k}_{3}\right)}{k^{6}} \frac{H^{6}}{M_{P}^{6}} \frac{e^{6 \pi \xi}}{\xi^{9}}
$$

Large nongaussianities in tensors:

$<h h h>\sim<h>^{3 / 2}$ 


\section{Constraints from the scalar sector?}

If $\phi$ is the inflaton, then gauge modes backreact on it, contributing to its three-point function

\section{- \\ NONGAUSSIANITIES}

Planck constraints on $f_{N L}$ equil

$$
\Delta \chi<<1
$$

Parity violation not detectable in this simplest model 


\section{One possible (and inelegant) way out:}

\section{MANY, MANY GAUGE FIELDS}

\section{Contributions to $f_{N L}$ add incoherently. With $\sim O\left(10^{3}\right)$ gauge fields $f_{N L}$ safely small}
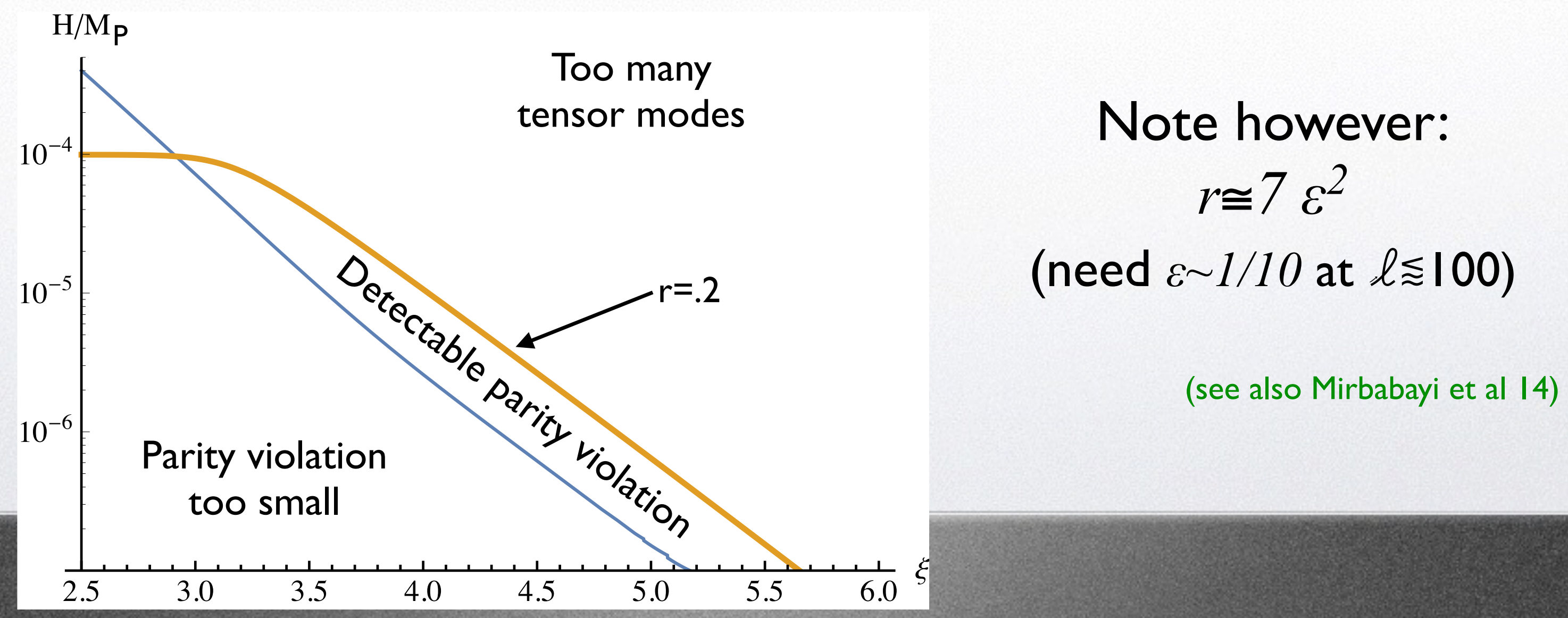


\section{Incidentally:}

\section{IN THIS SCENARIO RELATION BETWEEN $r$ AND $H^{2} / M_{P}^{2}$ DOES NOT HOLD!}

Senatore Silverstein Zaldarriaga I I

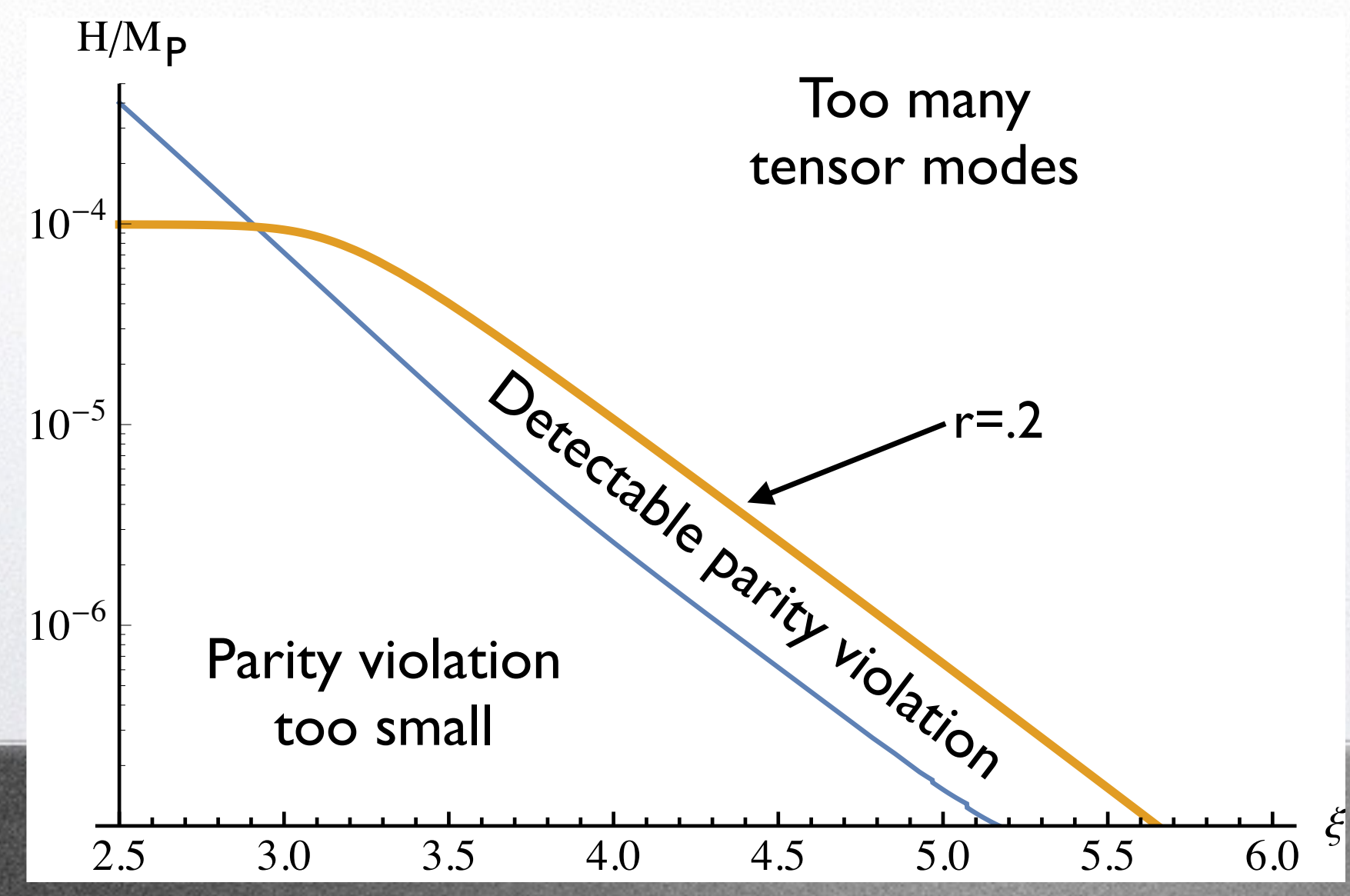




\section{also note}

\section{Tensor 3-pt function suppressed as scalar 3-pt function}

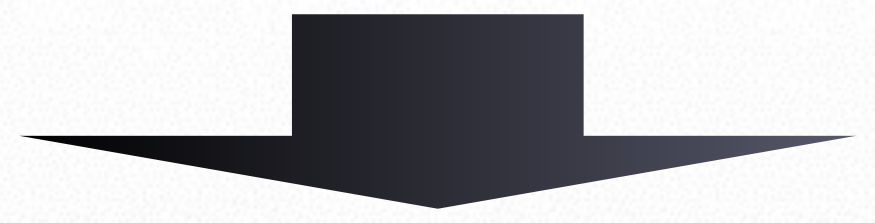

Parity-violating spectra, but gaussian tensors 


\section{One more possible way out: $\phi$ IS NOT THE INFLATON}

(let us call it $\sigma$ here)

however...

during super-horizon evolution isocurvature $\sigma$ modes mix with curvature perturbation:

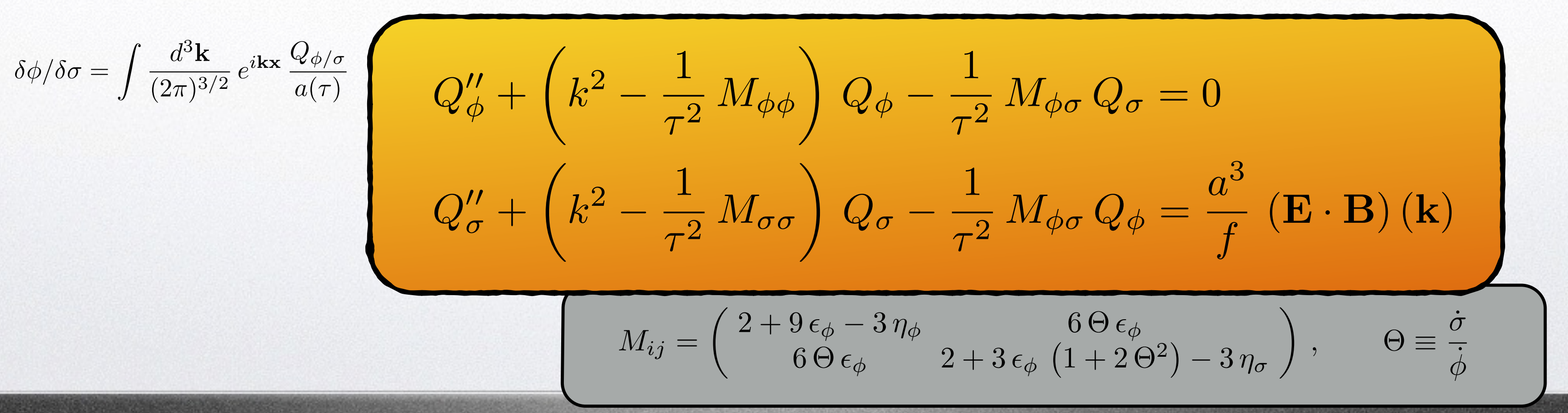




\section{One more possible way out: $\phi$ IS NOT THE INFLATON}

(let us call it $\sigma$ here)

Even if $\sigma \neq \phi$, contribution to $\xi$ same as in case where $\phi$ is the inflaton, times $\varepsilon$ times $\Delta N$

\# of efoldings of rolling of $\sigma$ 


\section{One more possible way out: $\phi$ IS NOT THE INFLATON}

(let us call it $\sigma$ here)

\section{Even if $\sigma \neq \phi$, contribution to $\zeta$}

same as in case where $\phi$ is the inflaton, times $\varepsilon$ times $\Delta N$

can have $\Delta N<<60$ :

assuming induced scalar perturbations < standard ones,

$$
r \lesssim O(f e w) / \Delta N^{2}
$$

Note: constraints from $f_{\mathrm{NL}}$ weaker as nongaussian contribution is strongly scale-dependent! 


\title{
Conclusion (partial)
}

Existence proof of

\author{
Parity violation
}

$+$

Large tensor nongaussianities

$=$

Interesting phenomenology in the CMB.

(some work with model building needed, though) 


\section{Intermezzo}

A "natural" coupling that might lead to nonvanishing $\langle E B\rangle$ and $\langle T B\rangle$ is

$$
\delta \mathcal{L}=\frac{\phi}{f^{\prime}} \epsilon_{\alpha \beta \gamma \delta} R_{\mu \nu}^{\alpha \beta} R_{\gamma \delta}^{\mu \nu}
$$

however... 
Action for tensor modes in theory with $\phi R \tilde{R}$

$$
\begin{array}{r}
\mathcal{S}=\sum \frac{1}{2} \int d \tau \frac{d^{3} k}{(2 \pi)^{3}} A_{\lambda}\left(\left|h_{\lambda}^{\prime}\right|^{2}-k^{2}\left|h_{\lambda}\right|^{2}\right) \\
A_{\lambda}=1-\lambda \frac{k}{a} \frac{\dot{\phi}}{2 f^{\prime} M_{P}^{2}}
\end{array}
$$

for $k$ too large one of the modes is strongly coupled and/or a ghost

if we choose parameters so to stay away from strongly coupled regime, then effect on tensor modes is too weak 


\section{More fun with GWs...}

...back to the case where the inflaton is directly coupled to the gauge field... 


\section{Inflationary}

$$
\begin{aligned}
& \mathcal{P}_{R}(\mathbf{k})= \frac{H^{2}}{\pi^{2} M_{P}^{2}}\left(1+9 \times 10^{-}\left(\frac{H^{2}}{M_{P}^{2}} \frac{e^{4 \pi \xi}}{\xi^{6}}\right)\right. \\
& \mathcal{P}_{L}(\mathbf{k})= \frac{H^{2}}{\pi^{2} M_{P}^{2}}\left(1+2 \times 10^{-}\left(\frac{H^{2}}{M_{P}^{2}} \frac{e^{4 \pi \xi}}{\xi^{6}}\right)\right) \\
& \xi \text { increases during inflation } \\
& \xi \equiv \frac{\dot{\phi}}{2 f H} \gtrsim 1
\end{aligned}
$$

GWs produced towards the end of inflation (i.e. at smaller scales) have larger amplitude might be detected by advanced LIGO! 
Prospects of direct detection of GWs of inflationary origin

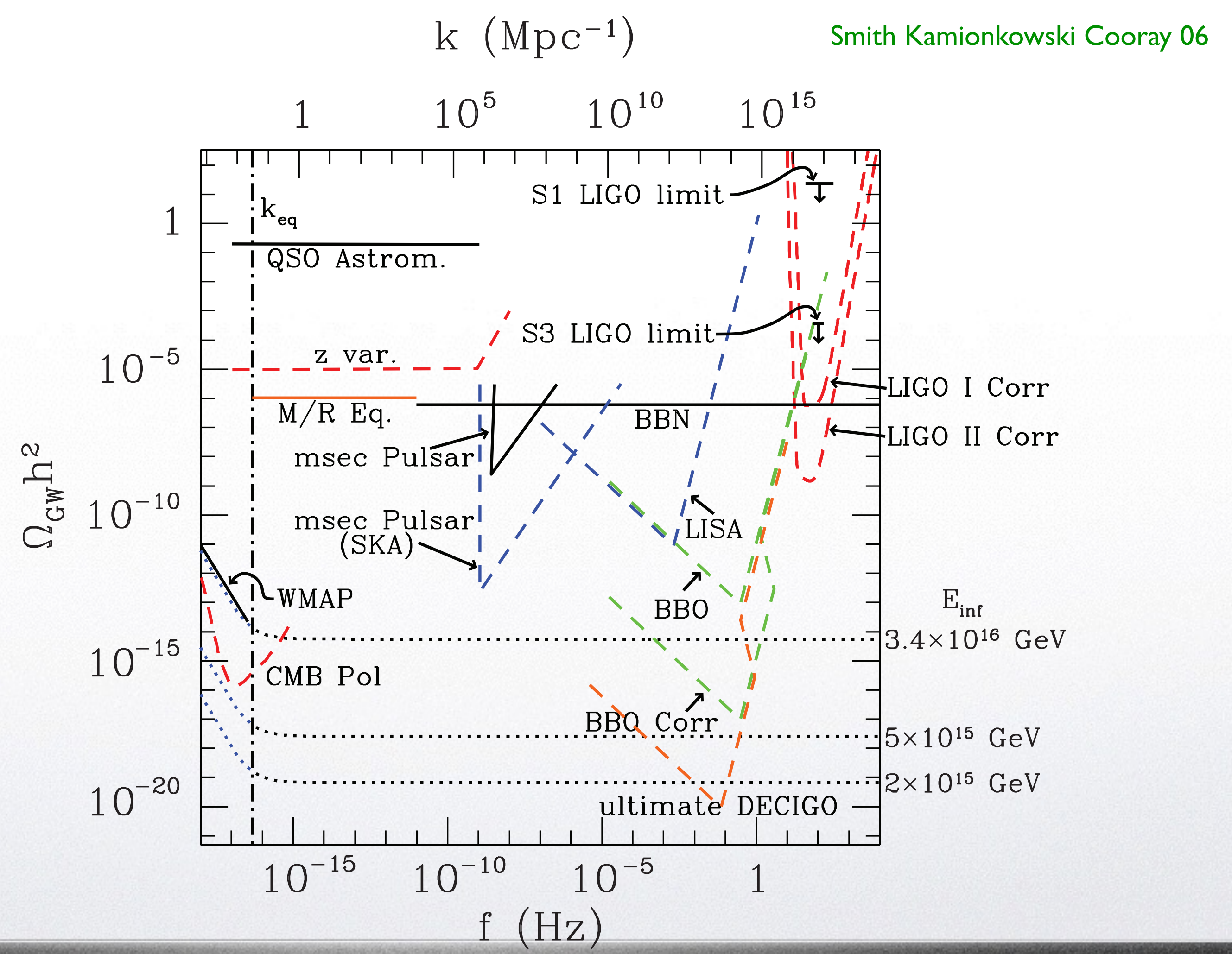



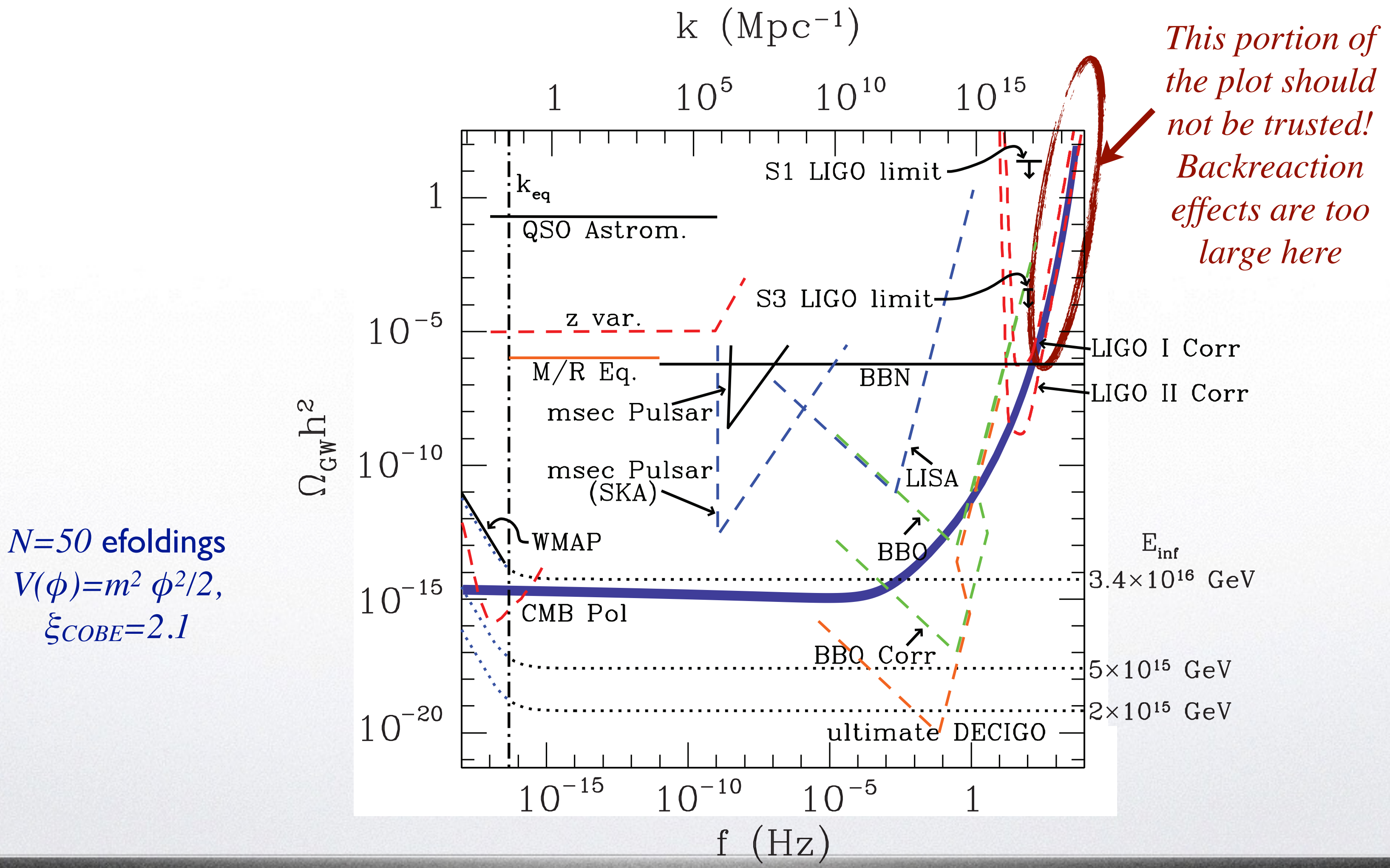


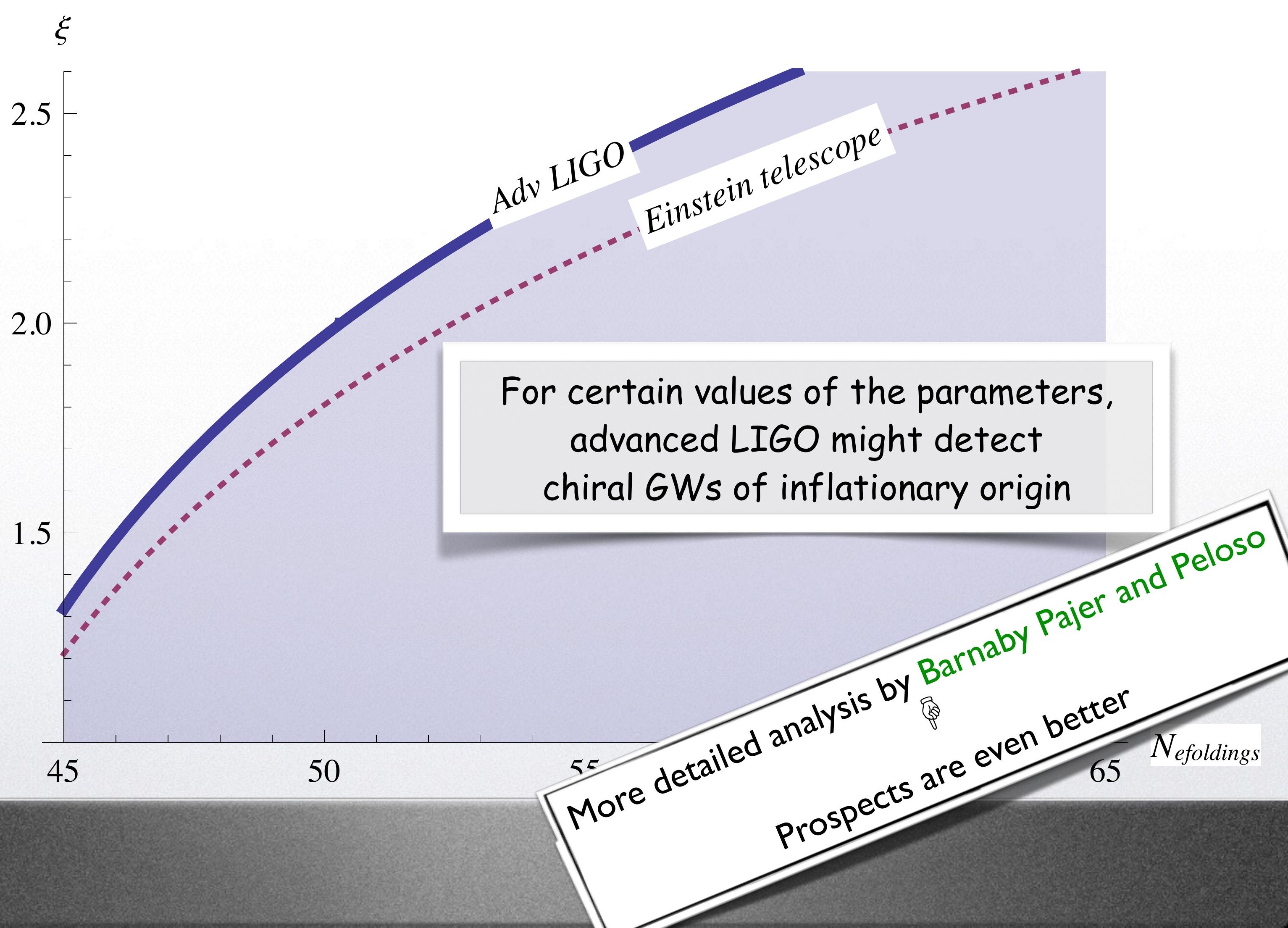




\section{A few comments}

These tensor modes would be chiral! Crowder et al 12

The GWs produced this way should be strongly nongaussian Thrane 12

Signal might correlate with nongaussianities at CMB/ LSS scales

Large and nongaussian fluctuations at the end of inflation might generate primordial $\mathrm{BHs}$

Linde and Pajer I3 


\section{And by the way....}

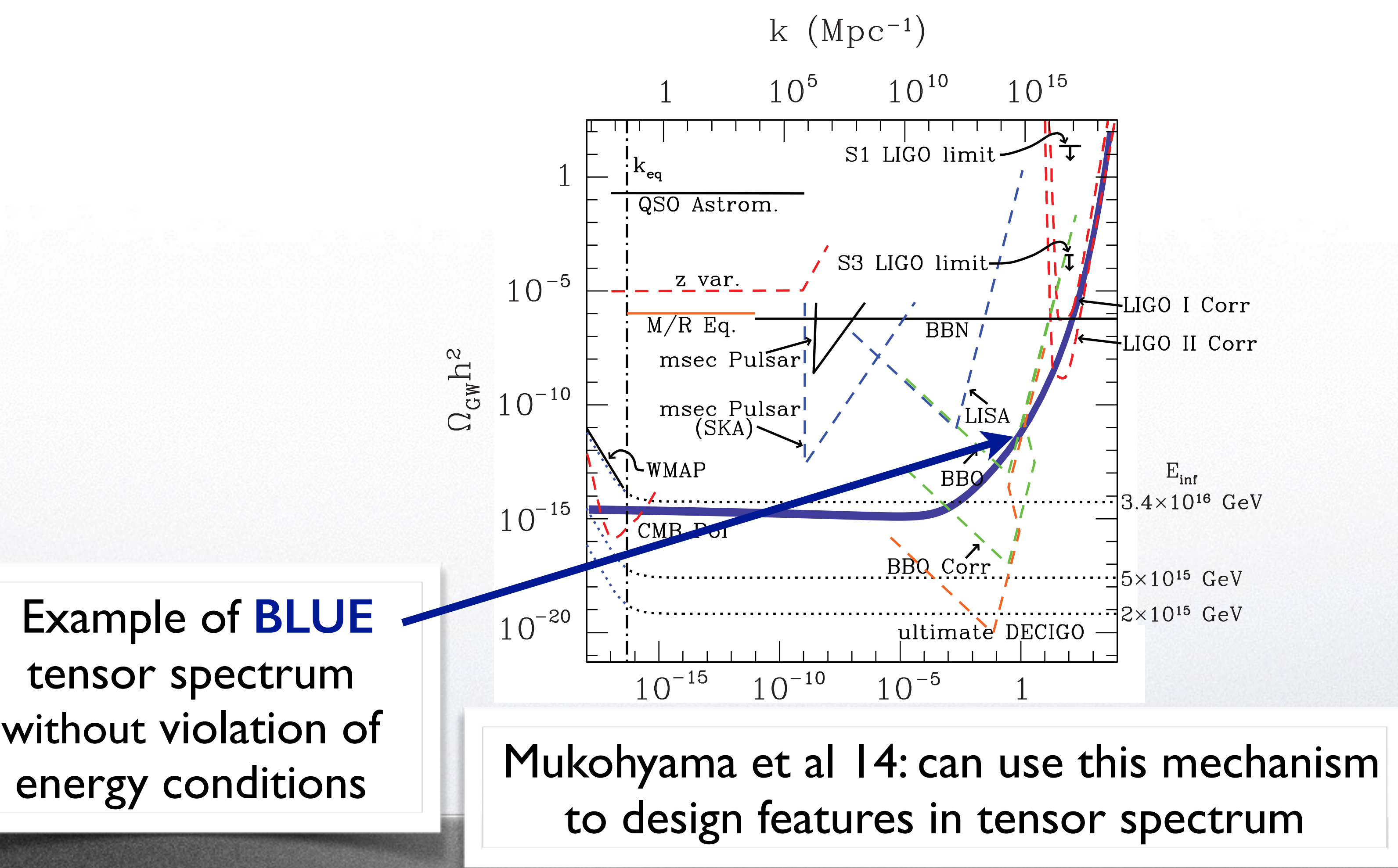




\section{Conclusion}

Tensors can have a very rich phenomenology 